\title{
Correlations between three-dimensional speckle-tracking echocardiography-derived left atrial functional parameters and aortic stiffness in healthy subjects - Results from the MAGYAR-Healthy Study
}

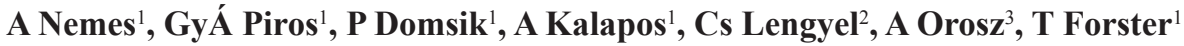 \\ 12nd Department of Medicine and Cardiology Centre, Medical Faculty, Albert Szent-Györgyi Clinical Center, \\ University of Szeged, Szeged, Hungary \\ ${ }^{2} 1$ st Department of Medicine, Medical Faculty, Albert Szent-Györgyi Clinical Center, \\ University of Szeged, Szeged, Hungary \\ ${ }^{3}$ Department of Pharmacology and Pharmacotherapy, Medical Faculty, Albert Szent-Györgyi Clinical Center, \\ University of Szeged, Szeged, Hungary
}

Left atrial (LA) distension has been demonstrated to be linked with aortic stiffness in different patient populations. Three-dimensional (3D) speckle-tracking echocardiography (STE) seems to be a promising tool for volumetric and functional evaluation of the LA. The aim of the present study was to determine whether correlations exist between 3DSTE-derived LA volume-based and strain parameters characterizing all phasic functions of the LA and echocardiographic aortic elastic properties in healthy subjects. The study included 19 healthy volunteers (mean age: $37.9 \pm 11.4$ years, 11 men) who had undergone complete two-dimensional (2D) Doppler transthoracic echocardiography extended with the assessment of aortic elastic properties and 3DSTE. Results: None of LA volumes correlated with echocardiographic aortic elastic properties. Active atrial stroke volume correlated with aortic stiffness index (ASI, $r=0.45, p=0.05$ ). None of other volume-based functional properties significantly correlated with aortic stiffness parameters. Global peak 3D strain correlated with aortic strain $(r=-0.46, p=0.05)$. Global radial pre-atrial contraction strain correlated with ASI $(r=-0.49, p=0.04)$ and $\operatorname{AS}(r=-0.50, p=0.04)$. Conclusions: Correlations exist between 3DSTE-derived LA functional parameters and eschocardiographic aortic elastic properties in healthy subjects.

Keywords: aortic, distensibility, echocardiography, function, left atrium, speckle-tracking, stiffness, threedimensional

There is a strong interplay between vascular and cardiac mechanics including arterialventricular coupling. Left ventricular (LV) remodeling is a powerful determinant of left atrial (LA) size, therefore arterial stiffness could influence LA dimensions, as well $(5,6,12)$. Arterial stiffness was found to be associated with LA size in different patient populations (12). Three-dimensional (3D) speckle-tracking echocardiography (STE) seems to be an optimal choice for non-invasive quantification of LV and LA volumes and deformation parameters by $3 \mathrm{D}$ volumetric and strain analysis $(1,4,7,8,11,13,15,17-19,22)$. The aim of the present study was to determine whether correlations exist between 3DSTE-derived LA volume-based and strain parameters characterizing all phasic functions of the LA and echocardiographic aortic elastic properties in healthy subjects.

Corresponding author: Attila Nemes, MD, PhD, DSc, FESC

2nd Department of Medicine and Cardiology Center, Medical Faculty

Albert Szent-Györgyi Clinical Center, University of Szeged

Semmelweis utca 6, P.O. Box 427, H-6725 Szeged, Hungary

Fax:+36-62-544568; Phone: +36-62-545220; E-mail: nemes.attila@med.u-szeged.hu 


\section{Materials and Methods}

\section{Patient population}

The study included 19 healthy volunteers (mean age: $37.9 \pm 11.4$ years, 11 men) who had undergone complete two-dimensional (2D) Doppler transthoracic echocardiography extended with echocardiographic aortic elastic properties assessments. 3DSTE has also been performed following 2D echocardiography in all cases. None of the subjects had any known disease which could have affected results. All subjects have been included in the MAGYAR- Healthy Study (Motion Analysis of the heart and Great vessels bY three-dimensionAl speckletRacking echocardiography in Healthy subjects). The study aimed to evaluate diagnostic and prognostic significance of 3DSTE-derived volumetric, strain, rotational, dyssynchrony, etc. parameters in healthy cases ('magyar' means 'Hungarian' in the Hungarian language). Informed consent was obtained from each patient and the study protocol conformed to the ethical guidelines of the 1975 Declaration of Helsinki, as reflected in a priori approval by the institution's human research committee (23).

\section{Two-dimensional echocardiography}

Standard 2D echocardiographic imaging was performed with the patient in the left lateral decubitus position using a commercially available Toshiba Artida ${ }^{\mathrm{TM}}$ echocardiography equipment (Toshiba Medical Systems, Tokyo, Japan) in the tissue harmonic mode. 2D echocardiographic images were obtained using the PST-30SBP (1-5 MHz) phased-array transducer in parasternal and apical 4-chamber $(\mathrm{AP} 4 \mathrm{CH})$ and 2-chamber $(\mathrm{AP} 2 \mathrm{CH})$ views. Special care was taken to avoid foreshortening during measurements. LV dimensions, volumes, ejection fraction and LA dimension were measured, while presence of valvular regurgitations and stenoses were excluded by Doppler echocardiography in all cases (21). All echocardiographic measurements were averaged from 3 beats.

\section{Measurement of blood pressure values}

Systolic (SBP) and diastolic blood pressure (DBP) values were estimated by a mercury cuff sphygmomanometer following 10 min of rest on the right arm in the supine position (16). The first Korotkoff sound for at least two consecutive heart beats was considered the SBP, while disappearance of fifth Korotkoff sound proved to be the DBP. Coffeinated drinks like coffee, tea, or other types of beverages, and cigarettes were not used or ingested from half and hour before the blood pressure measurements. Data were taken as the average of three consecutive measurements.

\section{Evaluation of aortic stiffness parameters}

Systolic and diastolic ascending aortic diameters (SD and DD, respectively) were recorded in M-mode echocardiography at a level of 3-4 $\mathrm{cm}$ above the aortic valve from a parasternal long-axis view as described in more details in the literature $(16,20)$ (Fig. 1). The SD and DD were considered at the time out of maximum aortic anterior motion and at the peak of QRS complex, respectively. All measurements were repeated 3 times, and average data have been given. Echocardiographic aortic elastic properties were calculated using the following equations:

[1] Aortic strain $[\mathrm{AS}]=(\mathrm{SD}-\mathrm{DD}) / \mathrm{DD}$

[2] Aortic stiffness index [ASI] $=\ln (\mathrm{SBP} / \mathrm{DBP}) /[(\mathrm{SD}-\mathrm{DD}) / \mathrm{DD}]$, where 'ln' is the natural logarithm

[3] Aortic distensibility $[\mathrm{AD}]=2 \times(\mathrm{SD}-\mathrm{DD}) /[(\mathrm{SBP}-\mathrm{DBP}) \times \mathrm{DD}]$ 


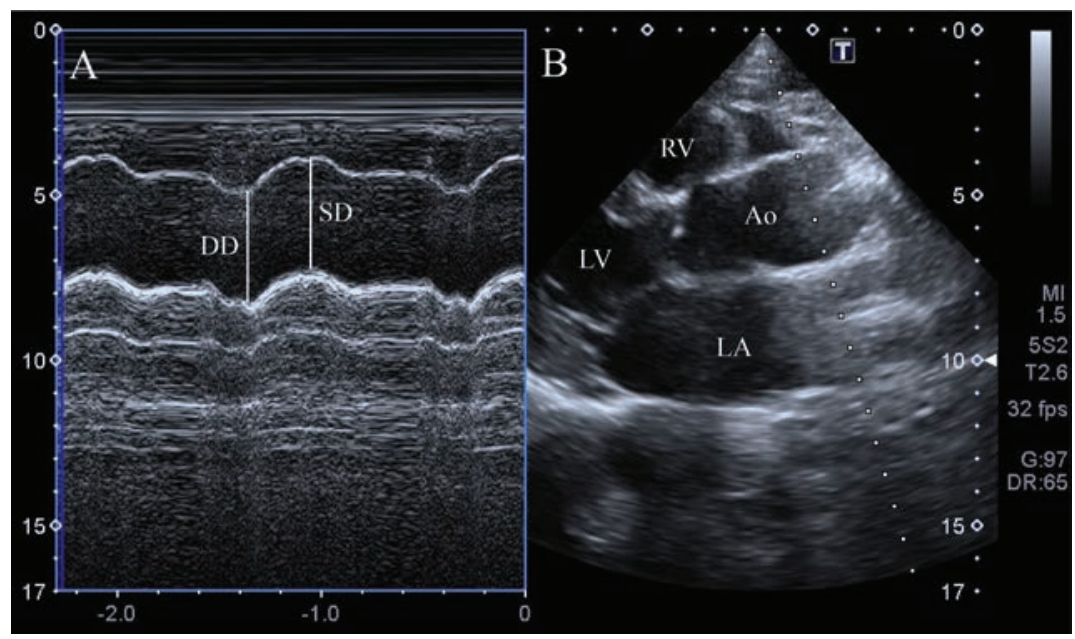

Fig. 1. Measurements of systolic (SD) and diastolic (DD) diameters of the ascending aorta (A) are shown on the M-mode tracing obtained at a level $3 \mathrm{~cm}$ above the aortic valve (B) at parasternal long-axis view.

Abbreviations: $\mathrm{LV}=$ left ventricle, $\mathrm{RV}=$ right ventricle, $\mathrm{LA}=$ left atrium, Ao = ascending aorta

\section{DSTE-derived volumetric measurements}

All patients underwent 3D echocardiographic acquisitions immediately after 2D echocardiographic study from the same apical window using the fully sampled PST-25SX matrix-array transducer (Toshiba Medical Systems, Tokyo, Japan) with 3DSTE capability (19). During acquisitions full volume mode was used in which six wedge-shaped subvolumes were acquired over six consecutive cardiac cycles during a single breath-hold. If there was an opportunity the sector width was decreased as much as possible to improve temporal and spatial image resolutions. Pyramid-shape 3D datasets were analysed using 3D Wall Motion Tracking software version 2.5 (Toshiba Medical Systems, Tokyo, Japan) by experienced investigators (AN, PD). AP4CH, AP2CH and 3 short-axis views at different levels of the LA (basal, midatrial, and superior LA regions) were automatically selected from the 3D pyramidal dataset (Fig. 2). In the AP4CH and $\mathrm{AP} 2 \mathrm{CH}$ views, the LA endocardial boundaries were manually traced by setting multiple reference points starting at the mitral valve level going toward the LA apex. Regarding to the literature LA appendage and pulmonary veins were not considered as the part of the LA cavity during 3DSTE assessments $(7,18)$. The epicardial border was adjusted manually or by setting a default thickness for the myocardium. After detection of the LA borders at the end-diastolic reference frame 3D endocardial surface was automatically reconstructed and tracked in 3D throughout the cardiac cycle. The user could manually adjust endocardial and epicardial LA surface when it was necessary. The following volumes have been calculated $(7,8,11,15,18)$ :

[1] maximum LA volume $\left(\mathrm{V}_{\max }\right)$ defined as the largest $\mathrm{LA}$ volume at end-systole just before mitral valve opening,

[2] minimum LA volume $\left(\mathrm{V}_{\min }\right)$ defined as the smallest $\mathrm{LA}$ volume at end-diastole before mitral valve closure,

[3] LA volume before atrial contraction $\left(\mathrm{V}_{\text {prea }}\right)$ defined at the last frame before mitral valve reopening or at the time of $\mathrm{P}$ wave on ECG at early diastole. 


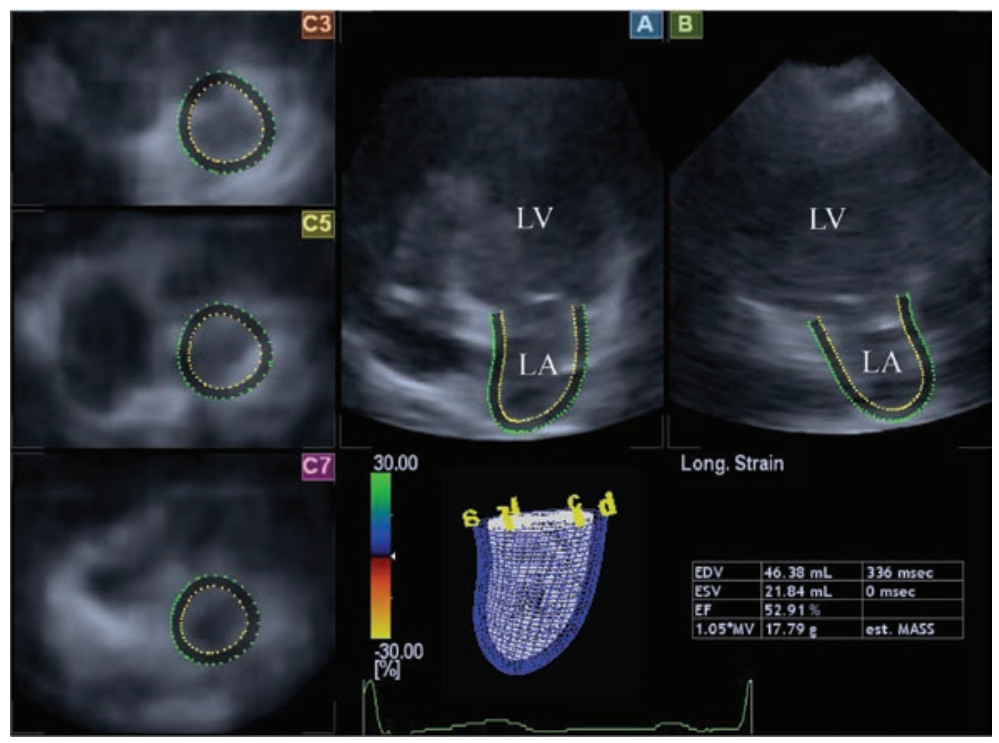

Fig. 2. Images from three-dimensional full-volume dataset showing left atrium in a patient with type 1 diabetes mellitus: (A) apical four-chamber view, (B) apical two-chamber view, (C3) parasternal short-axis view at basal, (C5) mid- and (C7) superior left atrial level. The semi-automated left atrial border definition and three-dimensional "wire" reconstruction of the left atrium based on three-dimensional speckle tracking echocardiographic analysis are also presented.

Abbreviations: $\mathrm{LA}=$ left atrium, $\mathrm{LV}=$ left ventricle

Several LA volume-based parameters characterizing each phasis of LA function were calculated from these volumes as demonstrated in Table I.

Table I. Calculation of left atrial stroke volumes and emptying fractions in different phasis of left atrial motion respecting cardiac cycle is presented

\begin{tabular}{|l|c|c|}
\hline \multicolumn{1}{|c|}{ Functions } & $\begin{array}{c}\text { Stroke volumes } \\
(\mathbf{m l})\end{array}$ & $\begin{array}{c}\text { Emptying fractions } \\
(\mathbf{\%})\end{array}$ \\
\hline Reservoir (Systole) & Total atrial SV & Total atrial $\mathrm{EF}=$ \\
& $\mathrm{V}_{\max }-\mathrm{V}_{\min }$ & Total atrial SV/ $\mathrm{V}_{\max }$ \\
\hline Conduit function (Diastole) & Passive atrial SV & Passive atrial $\mathrm{EF}=$ \\
& $\mathrm{V}_{\max }-\mathrm{V}_{\text {preA }}$ & Passive atrial SV/ $\mathrm{V}_{\max }$ \\
\hline Active contraction (Diastole) & Active atrial SV & Active atrial $\mathrm{EF}=$ \\
& $\mathrm{V}_{\text {preA }}-\mathrm{V}_{\min }$ & Active atrial SV/V \\
& & \\
\hline
\end{tabular}

Abbreviations: $\mathrm{EF}=$ emptying fraction, $\mathrm{SV}=$ stroke volume, $\mathrm{V}_{\max }=$ maximum left atrial volume, $\mathrm{V}_{\min }=$ minimum left atrial volume, $\mathrm{V}_{\text {preA }}=$ left atrial volume before atrial contraction

\section{DSTE-derived strain measurements}

From the same 3D echocardiographic dataset the following LA deformation parameters were calculated $(4,7,13,14)$ (Fig. 2):

[1] Longitudinal strain (strain in the direction parallel to the endocardial contour),

[2] Circumferential strain (fiber shortening along the circular perimeter, strain in the circumferential direction), 
[3] Radial strain (radially directed deformation, strain in the perpendicular direction),

[4] 3D strain (strain in the wall thickening direction, combination of radial, circumferential and longitudinal strains), and

[5] Area strain (ratio of endocardial area change during the cardiac cycle, percentage change in area).

Global and mean segmental peak and pre-atrial contraction LA strain parameters were calculated in each patient.

\section{Stastical analysis}

Statistical analyses were performed using the MedCalc software (MedCalc, Mariakerke, Belgium). All continuous variables are expressed as mean \pm standard deviation. Statistical significance was determined as a $P$ value of less than 0.05 for all tests. To compare continuous variables independent samples Student's $t$-test were used. Chi-square tests were used for comparison of categorical variables.

\section{Results}

Two-dimensional echocardiographic data

Routine 2D echocardiographic LV and LA data and aortic elastic properties are summarized in Table II.

Table II. Two-dimensional echocardiographic data and aortic elastic properties of subjects

\begin{tabular}{|l|c|}
\hline & Data \\
\hline Left ventricular diastolic diameter $(\mathrm{mm})$ & $48.0 \pm 6.8$ \\
\hline Left ventricular systolic diameter $(\mathrm{mm})$ & $30.1 \pm 4.2$ \\
\hline Left ventricular diastolic volume $(\mathrm{ml})$ & $100.7 \pm 20.2$ \\
\hline Left ventricular systolic volume $(\mathrm{ml})$ & $34.8 \pm 11.0$ \\
\hline Interventricular septum $(\mathrm{mm})$ & $9.5 \pm 2.0$ \\
\hline Left ventricular posterior wall $(\mathrm{mm})$ & $9.5 \pm 2.3$ \\
\hline Left ventricular ejection fraction $(\%)$ & $65.7 \pm 7.0$ \\
\hline Systolic aortic diameter $(\mathrm{mm})$ & $30.3 \pm 3.6$ \\
\hline Diastolic aortic diameter $(\mathrm{mm})$ & $26.8 \pm 3.8$ \\
\hline Systolic minus diastolic aortic diameter $(\mathrm{mm})$ & $3.50 \pm 2.28$ \\
\hline Aortic strain & $0.13 \pm 0.09$ \\
\hline Aortic distensibility $\left(\mathrm{cm}^{2} /\right.$ dynes $\left.10^{-6}\right)$ & $4.58 \pm 3.21$ \\
\hline Aortic stiffness index & $5.17 \pm 3.45$ \\
\hline
\end{tabular}

Three-dimensional speckle-tracking echocardiographic data

3DSTE-derived LA volumes, volume-based functional properties and strain parameters are summarized in Tables III and IV.

Correlations (volumetric data vs. aortic elastic properties)

None of LA volumes correlated with echocardiographic aortic elastic properties. Active atrial stroke volume correlated with ASI $(r=0.45, p=0.05)$, while passive atrial stroke volume tended to be correlated with ASI $(r=-0.42, p=0.09)$. None of other volume-based functional properties correlated with any of aortic stiffness parameters. 
Table III. Comparison of 3DSTE-derived volumetric and volume-based functional left atrial parameters in patients with type 1 diabetes mellitus and controls

\begin{tabular}{|l|c|}
\hline & Data \\
\hline Calculated volumes $(\mathrm{ml})$ & $35.6 \pm 6.4$ \\
\hline Maximum left atrial volume $\left(\mathrm{V}_{\text {max }}\right)$ & $16.3 \pm 4.9$ \\
\hline Minimum left atrial volume $\left(\mathrm{V}_{\text {min }}\right)$ & $23.8 \pm 6.7$ \\
\hline Left atrial volume before atrial contraction $\left(\mathrm{V}_{\text {preA }}\right)$ & \\
\hline Stroke volumes $(\mathrm{ml})$ & $19.3 \pm 4.5$ \\
\hline Total atrial stroke volume & $11.8 \pm 4.7$ \\
\hline Passive atrial stroke volume & $7.5 \pm 3.2$ \\
\hline Active stroke volume & \\
\hline Emptying fractions $(\%)$ & $54.5 \pm 10.2$ \\
\hline Total atrial emptying fraction & $33.5 \pm 12.3$ \\
\hline Passive atrial emptying fraction & $31.4 \pm 9.2$ \\
\hline Active atrial emptying fraction & \\
\hline
\end{tabular}

Table IV. Comparison of 3DSTE-derived global and mean segmental peak and pre-atrial contraction left atrial strain parameters in healthy subjects

\begin{tabular}{|l|c|c|}
\hline & Peak & Pre-atrial contraction \\
\hline Global strain parameters & & $-8.5 \pm 8.3$ \\
\hline Radial strain (\%) & $-21.8 \pm 11.8$ & $10.7 \pm 11.4$ \\
\hline Circumferential strain (\%) & $28.7 \pm 10.0$ & $9.0 \pm 9.4$ \\
\hline Longitudinal strain (\%) & $24.2 \pm 6.6$ & $16.5 \pm 16.5$ \\
\hline Area strain (\%) & $57.7 \pm 17.6$ & $-5.3 \pm 5.4$ \\
\hline 3D strain (\%) & $-13.9 \pm 10.8$ & \\
\hline Mean segmental strain parameters & & $-8.6 \pm 5.5$ \\
\hline Radial strain (\%) & $-23.1 \pm 9.0$ & $13.3 \pm 10.0$ \\
\hline Circumferential strain (\%) & $36.6 \pm 12.4$ & $9.2 \pm 6.2$ \\
\hline Longitudinal strain (\%) & $31.6 \pm 6.7$ & $21.2 \pm 15.1$ \\
\hline Area strain (\%) & $74.5 \pm 23.2$ & $-6.7 \pm 5.0$ \\
\hline 3D strain (\%) & $-16.4 \pm 6.8$ & \\
\hline
\end{tabular}

Abbreviation: $3 \mathrm{D}=$ three-dimensional

Fig. 3. Schematic figure demonstrating volumetric and strain changes during all the three functions of the left atrium and their relation to the cardiac cyle

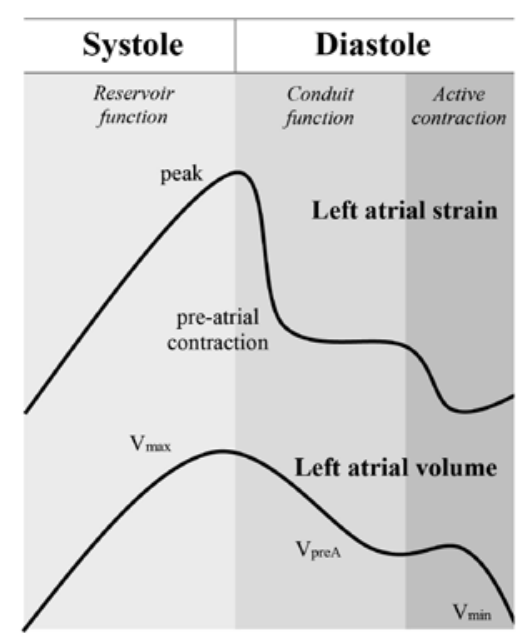


Correlations (peak strains vs. aortic elastic properties)

Global peak 3D strain correlated with aortic strain $(r=-0.46, p=0.05)$. Only tendentious correlations could be demonstrated between global radial peak strain and ASI $(r=-0.39$, $p=0.08)$ and AS $(r=-0.41, p=0.07)$ and between mean longitudinal peak strain and AS $(r=0.41, p=0.08)$.

Correlations (pre-atrial contraction strains vs. aortic elastic properties)

Global radial pre-atrial contraction strain correlated with ASI $(r=-0.49, p=0.04)$ and AS $(r=-0.50, p=0.04)$ and tended to be correlated with $\mathrm{AD}(r=0.43, p=0.07)$.

\section{Discussion}

To the best of authors' knowledge this is the first study in which correlations could be demonstrated between echocardiographic aortic elastic properties and 3DSTE-derived LA functional parameters in healthy subjects. 3DSTE is a new non-invasive clinical tool based on frame-by-frame tracking of speckle patterns created by interference of the ultrasound beam within the myocardial tissue in the 3D space $(1,22)$. 3DSTE has been found to be feasible for non-invasive quantification of LA volumes and functional properties allowing complex evaluation of LA phasic function during cardiac cycle which includes (2, 9) (Fig. 3):

[1] Reservoir function (LA inflow during LA systole),

[2] Conduit function (LA passive emptying during LV relaxation and diastasis, when blood transiting from the pulmonary veins to the LV during early diastole),

[3] Active contraction or booster pump function (LA active emptying, when LA works like an active contractile chamber that augments LV filling in late diastole).

There are several ways for functional assessment of LA including calculation of volumebased and strain parameters by 3DSTE as demonstrated before $(4,7,8,11,13,15,18)$. With these parameters detailed characterization of all three LA functions possible:

[1] Reservoir function by total atrial SV and total atrial EF together with global and mean segmental peak strain parameters,

[2] Conduit function by passive atrial SV and passive atrial EF, and

[3] Active contraction (booster pump) by active atrial SV and active atrial EF together with global and mean segmental pre-atrial contraction strain parameters.

It is known that due to large number of collagens and filaments, the normal aorta is working as an elastic artery. As a physiologic consequence of the reduction of aortic buffering (Windkessel) function, SBP increases and DBP decreases leading to increased LV afterload and impaired LV relaxation (3). In the present study most of functional LA parameters showing correlations with aortic elastic properties are characteristics of atrial booster pump function reflecting magnitude and timing of atrial contractility but is dependent on the degree of venous return, LV end-diastolic pressures and LV systolic reserve (9). Moreover, correlations were found between aortic elasticity and characteristics of LA reservoir and conduit functions, as well. Because of the close interplay between LA, LV remodeling and diastolic function, the relationship is not suprising. However, in the present study detailed analysis could be demonstrated between echocardiographic aortic elastic properties and all the LA phasic functions by 3DSTE-derived volume-based and strain parameters even in healthy subjects. 


\section{Limitation section}

The following important limitations should be mentioned when interpreting results:

[1] Due to lower temporal and spatial image resolutions the 3DSTE-derived image quality is mostly worse than that of 2D echocardiography.

[2] Despite 3DSTE seems to be an applicable technique for non-invasive estimation of LA volumes and functional properties, more comparative and validation studies with other methodologies are warranted $(11,15,18)$.

[3] At this moment 3DSTE-derived normal strain reference values has not been described and the results of the present study were somewhat different as compared to that of previous findings. It could be explained by methodological differences, but the effect of the age and other factors could also not be excluded $(4,7,13)$.

[4] LA appendage and pulmonary veins were excluded from evaluations.

[5] It is known that LA function could be deteriorated in different arrhytmologic disorders like in atrial fibrillation. However, all of the studied healthy subjects were in sinus rhythm.

[6] Theoretically higher grade of MR could affect LA function. However, none of the healthy subjects had $\geq$ grade $1 \mathrm{MR}$.

[7] Quantification of LV strains and rotational parameters by 3DSTE was not aimed in the present study.

[8] The blood pressure in the brachial artery and ascending aorta may be different which could theoretically affect our results. However, the presented non-invasive imaging technique has been validated against invasive methods in the evaluation of aortic stiffness parameters $(3,20)$.

[9] 3DSTE was performed in only a small population of healthy subjects.

\section{Conclusions}

Correlations exist between 3DSTE-derived functional LA parameters and echocardiographic aortic elastic properties in healthy subjects.

\section{Conflict of Interest}

None declared.

\section{REFERENCES}

1. Ammar KA, Paterick TE, Khandheria BK, Jan MF, Kramer C, Umland MM, Tercius AJ, Baratta L, Tajik AJ: Myocardial mechanics: understanding and applying three-dimensional speckle tracking echocardiography in clinical practice. Echocardiography 29, 861-872 (2012)

2. Barbier P, Solomon, SB, Schiller NB, Glantz SA: Left atrial relaxation and left ventricular systolic function determine left atrial reservoir function. Circulation 100, 427-436 (1999)

3. Belz GG: Elastic properties and Windkessel function of the human aorta. Cardiovasc. Drugs Ther. 9, 73-83 (1995)

4. Chadaide S, Domsik P, Kalapos A, Saghy L, Forster T, Nemes A: Three-dimensional speckle tracking echocardiography-derived left atrial strain parameters are reduced in patients with atrial fibrillation (Results from the MAGYAR-Path Study). Echocardiography 30, 1078-1083 (2013) 
5. Chae CU, Pfeffer MA, Glynn RJ, Mitchell GF, Taylor JO, Hennekens CH: Increased pulse pressure and risk of heart failure in the elderly. JAMA 281, 634-639 (1999)

6. Cioffi G, Mureddu GF, Stefenelli C, de Simone G: Relationship between left ventricular geometry and left atrial size and function in patients with systemic hypertension. J. Hypertens. 22, 1589-1596 (2004)

7. Domsik P, Kalapos A, Chadaide S, Sepp R, Hausinger R, Forster T, Nemes A: Three-dimensional speckle tracking echocardiography allows detailed evaluation of left atrial function in hypertrophic cardiomyopathy Insights from the MAGYAR-Path Study. Echocardiography 31, 1245-1252 (2014)

8. Domsik P, Kalapos A, Lengyel C, Orosz A, Forster T, Nemes A: Correlations between mitral annular and left atrial function as assessed by three-dimensional speckle-tracking echocardiography in healthy volunteers. Results from the MAGYAR-Healthy Study. Orv. Hetil. 155, 1517-1523 (2014) (In Hungarian)

9. Hoit BD: Left atrial size and function: Role in prognosis. J. Am. Coll. Cardiol. 63, 493-505 (2014)

10. Kalapos A, Domsik P, Forster T, Nemes A: Left ventricular strain reduction is not confined to the noncompacted segments in noncompaction cardiomyopathy - Insights from the three-dimensional speckle tracking echocardiographic MAGYAR-Path Study. Echocardiography 31, 638-643 (2014)

11. Kleijn SA, Aly MF, Terwee CB, van Rossum AC, Kamp O: Comparison between direct volumetric and speckle tracking methodologies for left ventricular and left atrial chamber quantification by three-dimensional echocardiography. Am. J. Cardiol 108, 1038-1044 (2011)

12. Lantelme P, Laurent S, Besnard C, Bricca G, Vincent M, Legedz L, Milon H: Arterial stiffness is associated with left atrial size in hypertensive patients. Arch. Cardiovasc. Dis. 101, 35-40 (2008)

13. Mochizuki A, Yuda S, Oi Y, Nishida J, Kouzu H, Muranaka A, Kokubu N, Shimoshige S, Hashimoto A, Tsuchihashi K, Watanabe N, Miura T: Assessment of left atrial deformation and synchrony by three-dimensional speckle-tracking echocardiography: comparative studies in healthy subjects and patients with atrial fibrillation. J. Am. Soc. Echocardiogr. 26, 165-174 (2013)

14. Mondillo S, Galderisi M, Mele D, Cameli M, Lomoriello VS, Zacà V, Ballo P, D’Andrea A, Muraru D, Losi M, Agricola E, D’Errico A, Buralli S, Sciomer S, Nistri S, Badano L; Echocardiography Study Group Of The Italian Society Of Cardiology (Rome, Italy): Speckle-tracking echocardiography: a new technique for assessing myocardial function. J. Ultrasound Med. 30, 71-83 (2011)

15. Nagaya, M., Kawasaki, M., Tanaka, R., Onishi N, Sato N, Ono K, Watanabe T, Minatoguchi S, Miwa H, Goto Y, Hirose T, Arai M, Noda T, Watanabe S, Minatoguchi S: Quantitative validation of left atrial structure and function by two-dimensional and three-dimensional speckle tracking echocardiography: A comparative study with three-dimensional computed tomography. J. Cardiol. 62, 188-194 (2013)

16. Nemes A, Geleijnse ML, Forster T, Soliman OI, Ten Cate FJ, Csanady M: Echocardiographic evaluation and clinical implications of aortic stiffness and coronary flow reserve and their relation. Clin. Cardiol. 31, 304-309 (2008)

17. Nemes A, Kalapos A, Domsik P, Forster T: Three-dimensional speckle-tracking echocardiography - a further step in the non-invasive three-dimensional cardiac imaging. Orv. Hetil. 153, 1570-1577 (2012) (in Hungarian)

18. Nemes A, Domsik P, Kalapos A, Lengyel C, Orosz A, Forster T: Comparison of three-dimensional speckle tracking echocardiography and two-dimensional echocardiography for evaluation of left atrial size and function in healthy volunteers (Results from the MAGYAR-Healthy Study). Echocardiography 31, 865-871 (2014)

19. Nesser HJ, Mor-Avi V, Gorissen W, Weinert L, Steringer-Mascherbauer R, Niel J, Sugeng L, Lang RM: Quantification of left ventricular volumes using three-dimensional echocardiographic speckle tracking: comparison with MRI. Eur. Heart J. 30, 1565-1573 (2009)

20. Stefanadis C, Stratos C, Boudoulas H, Kourouklis C, Toutouzas P: Distensibility of the ascending aorta: comparison of invasive and non-invasive techniques in healthy men and in men with coronary artery disease. Eur. Heart J. 11, 990-996 (1990)

21. Teichholz LE, Cohen MV, Sonnenblick EH, Gorlin R: Study of left ventricular geometry and function by B-scan ultrasonography in patients with and without asynergy. N. Engl. J. Med. 291, 1220-1226 (1974)

22. Urbano-Moral JA, Patel AR, Maron MS, Arias-Godinez JA, Pandian NG: Three-dimensional speckle-tracking echocardiography: Methodological aspects and clinical potential. Echocardiography 29, 997-1010 (2012)

23. World Medical Association Declaration of Helsinki, Ethical Principles for Medical Research Involving Human Subjects. http:// www.wma.net/en/30publications/10policies/b3/index.html 